\title{
Effects of Packing Density on the Segregative Behaviors of Granular Systems
}

\author{
C. R. K. Windows-Yule, ${ }^{1, *}$ T. Weinhart, ${ }^{2}$ D. J. Parker, ${ }^{1}$ and A. R. Thornton ${ }^{3}$ \\ ${ }^{1}$ School of Physics and Astronomy, University of Birmingham, Edgbaston, Birmingham B15 2TT, United Kingdom \\ ${ }^{2}$ Multiscale Mechanics (MSM), Department of Mechanical Engineering, University of Twente, \\ P.O. Box 217, 7500 AE Enschede, The Netherlands \\ ${ }^{3}$ Multiscale Mechanics (MSM) and Mathematics of Computational Science (MaCS), (MESA $\left.{ }^{+}\right)$, CTW, University of Twente, \\ P.O. Box 217, 7500 AE Enschede, The Netherlands
}

(Received 27 September 2013; published 4 March 2014)

\begin{abstract}
We present results concerning the important role of system packing in the processes of density- and inelasticity-induced segregation in vibrofluidized binary granular beds. Data are acquired through a combination of experimental results acquired from positron emission particle tracking and simulations performed using the discrete particle method. It is found that segregation due to inelasticity differences between particle species is most pronounced in moderately dense systems, yet still exerts a significant effect in all but the highest density systems. Results concerning segregation due to disparities in particles' material densities show that the maximal degree to which a system can achieve segregation is directly related to the density of the system, while the rate at which segregation occurs shows an inverse relation. Based on this observation, a method of minimizing the time and energy requirements associated with producing a fully segregated system is proposed.
\end{abstract}

DOI: 10.1103/PhysRevLett.112.098001

Introduction.-Granular materials, systems comprising multiple macroscopic objects, display many phenomena unobserved in "normal," molecular materials [1]. For instance, a mechanically excited granular system containing two or more distinct "species" of particle may spontaneously separate into its individual constituents [2]. This granular segregation can be driven by various dissimilarities between particles, including differences in density [3], size [4], shape [5], or inelasticity [6,7]. An understanding of granular segregation is of great importance, due partly to its significant role in various industrial processes [8]. This Letter examines the segregative processes of particles differing in their densities and/or elastic properties for a wide range of packing fractions, encompassing both the high-density situation and the comparatively lessresearched highly fluidized regime. Analysis is performed using a combination of experimental results obtained using positron emission particle tracking (PEPT) and discrete particle simulations [9-12].

Experimental details.-The experimental system comprises a granular bed of $N=1000$ spherical particles of diameter $d=5 \mathrm{~mm}$, housed in a square-based cuboidal container. The large $d$ means that interstitial air effects, previously shown to influence segregation [13-15], may be neglected [16,17]. Particles with various densities $\rho$ and elasticities $\epsilon$ are combined to give a variety of bidisperse systems. For each system, $N_{\alpha}=N_{\beta}=500$, where $\alpha$ and $\beta$ denote individual particle species. The system is vibrated sinusoidally in the vertical direction, exciting the particles. The amplitude $A$ and frequency $f$ of oscillations are, respectively, $0.862 \mathrm{~mm}$ and $70 \mathrm{~Hz}$, giving a dimensionless
PACS numbers: 45.70.Mg, 47.57.Gc, $81.05 . \mathrm{Rm}, 83.80 . \mathrm{Fg}$

acceleration $\Gamma=\left(\left(4 \pi^{2} f^{2} A\right) / g\right)=17$ and shaking strength parameter $S=\left(A^{2} \omega^{2} / d g\right)$, where $g$ is the acceleration due to gravity. Although the current work explores the high shaking strength limit, making $S$ the relevant energy-input control parameter, the use of fixed driving parameters ensures that both $\Gamma$ and $S$ are held constant $[18,19]$. The appropriate control parameter for dissipation is $F_{d}=$ $H(1-\epsilon)$ [20], where $H$ is the static bed height normalized by $d$. Containers of width $W \in(40 \mathrm{~mm}, 140 \mathrm{~mm})$ are used, producing variation in $H$, specifically $H \in(1.3,15.6)$. Thus, the system's bulk packing fraction $\eta$ can be altered while maintaining constant driving and a consistent $N . \eta$ is defined as the average packing fraction calculated over a vertical height corresponding to the dynamic bed height $\tilde{H}$, equal to twice the vertical center of mass of the excited bed. For binary systems, $\epsilon$ is taken as the geometric average of the species' individual values. The relationship is found to hold over the entire range of $H, \epsilon$, and $\rho$ discussed in this Letter. Interestingly, the relation shows no significant $\rho$ dependence, as illustrated in Fig. 1(b), which shows simulational data alongside curves corresponding to the proposed empirical relation. This independence is thought to be due to the large $\Gamma$ values used. The system is lubricated by $0.5 \mathrm{~g}$ of graphite powder [21,22]. The corresponding reduction in friction isolates, as far as possible, behaviors associated solely with density and elasticity and also frustrates convection in denser systems [23]. Data are extracted using PEPT, whereby the timeaveraged behavior of a single tracer particle can, for an ergodic system, be used to extrapolate a variety of timeaveraged quantities pertaining to the system as a whole. 


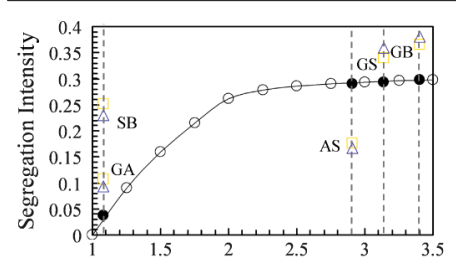

(a)

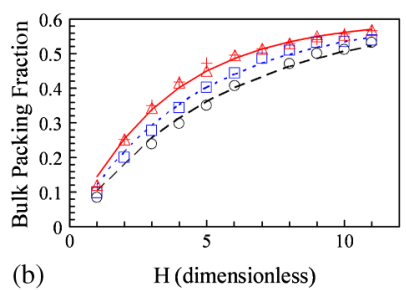

FIG. 1 (color online). (a) Segregation intensity $I_{S}$ as a function of density ratio $\rho_{H} / \rho_{L}$ for $H=2.5$. Definitions of the symbols used are given in the main text. (b) Variation of packing fraction with $H$ for $\epsilon$ and $\rho$ values corresponding to glass (circles and dashed line), brass (triangles and solid line), and binary brass or glass (squares and dotted line) systems. Crosses represent simulations implementing the elasticity of brass with a reduced density $\left(\rho=2500 \mathrm{~kg} \mathrm{~m}^{-3}\right)$, demonstrating a seeming mass independence.

PEPT is nonintrusive, and images in three dimensions, to a spatial resolution of $1 \mathrm{~mm}$ and a temporal resolution of the order of milliseconds. For full information regarding PEPT and its application to bidisperse systems, please refer to Refs. [24,25] and [26,27], respectively.

Simulation details.-Simulations are performed using the MerCuRyDPM code, implementing the experimental values of $f, A, N, d, H$, and $\rho$. Values of the interparticle coefficient of restitution for single species collisions $\epsilon_{\alpha \alpha}$ are extrapolated from values determined experimentally by Feitosa and Menon [28]. Specific values of $\rho$ and $\epsilon_{\alpha \alpha}$ can be seen in Table I. Since these effective elasticities [28] account for translational energy losses to all sources, including conversion of translational to rotational kinetic energy, the frictional coefficient $\mu$ is set to 0 . As with the addition of a lubricant in the experimental system, the suppression of specific frictional effects allows the role of inelasticity to be more clearly examined. For interspecies collisions, the dissipative coefficient $\epsilon_{\alpha \beta}$ is taken simply as the geometric average of the relevant single-species values [29]. Particle-wall collisions are modeled as inelastic, with coefficient of restitution $\epsilon_{w}=0.59$ [33]. For full details regarding MERcuryDPM, please see the Supplemental Material [35].

Results and discussion.-The extent of segregation can be quantified using the steady-state segregation intensity $I_{s}$, as defined in [36]. For the system under investigation, $I_{s}$ can be calculated by dividing the experimental volume into

TABLE I. Effective elasticities $\epsilon_{\alpha \alpha}$ and material densities $\rho$ for the various particles used in experiment.

\begin{tabular}{lcc}
\hline \hline Particle material & $\rho\left(\mathrm{kg} \mathrm{m}^{-3}\right)$ & $\epsilon_{\alpha \alpha}$ \\
\hline Glass (G) & 2500 & 0.83 \\
Aluminum (A) & 2700 & 0.69 \\
Steel (S) & 7850 & 0.79 \\
Brass (B) & 8500 & 0.61 \\
\hline \hline
\end{tabular}

a series of $N_{c}$ equally sized cells in all spatial dimensions. For each cell, the local number fraction of a single component $\varphi_{i}=\eta_{i} /\left(\eta_{i}+\eta_{j}\right)$ is calculated and compared to the system mean $\varphi_{m}=0.5$ and $I_{s}$ determined as

$$
I_{s}=\left[\frac{\sum_{i=1}^{i=N_{c}}\left(\varphi_{i}-\varphi_{m}\right)^{2}}{N_{c}}\right]^{1 / 2} .
$$

$I_{s}=0$ for a perfectly mixed system and 0.5 for a complete segregation. Figure 1 shows experimental (triangles) and simulational (squares) values of $I_{s}$ for various material combinations in a system of $H=2.5$. Specific combinations are denoted using the abbreviations given in Table I. Although strong agreement between experiment and simulation is observed, the simultaneous variation of elasticity and density prevents the observation of individual trends. Shown also is the expected trend for segregation driven purely density differences $\left(\epsilon_{\alpha \alpha}=\epsilon_{\beta \beta}=0.91\right)$. For the data sets represented by open circles, the density of the light component is fixed at $2500 \mathrm{~kg} \mathrm{~m}^{-3}$ and that of the heavy component varied. Filled circles represent the experimental values of density, as given in Table I. The collapse of these points onto a single trend implies that the average material density of a system does not significantly influence its segregative behavior. The divergence from this trend for the case of differing elasticities illustrates the profound impact of inelasticity effects on the segregation of highly fluidized systems. This pronounced impact of $\epsilon_{\alpha \alpha} / \epsilon_{\beta \beta}$ persists even at large values of $\rho_{H} / \rho_{L}$, the ratio of densities for the light and heavy components of the system, where one might expect density effects to dwarf those due to inelasticity-a noteworthy observation. The magnitude of the effect of $\epsilon_{\alpha \alpha} / \epsilon_{\beta \beta}$ on segregative behavior is perhaps best illustrated by comparing a system of steel and glass spheres to a system of steel and aluminum spheres. If we consider only segregation driven by differences in particle density, then simulations predict $I_{s}$ to vary by a factor of 1.01 between the two cases. When inelasticity is considered, however, this variation increases to more than a factor of 2 . The reasoning behind this significant discrepancy is simple - in the steel and glass case, the heavier component is also more dissipative, so the two segregative processes act in unison, creating greater segregation than would be expected due to density differences alone. For the steel and aluminum case, however, the processes act in opposition, resulting in an overall decrease in the degree of segregation.

A second notable feature of Fig. 1 is, for solely densitydriven segregation, an apparent tendency toward an asymptotic value at large $\rho_{H} / \rho_{L}$ that is considerably below $I_{s}=0.5$. The implication here of a maximal $I_{s}$ for relatively dilute systems is understandable - the presence of voids between particles introduces a probability of light particles "falling through" the system, preventing complete segregation [37,38]. It is interesting to note that the introduction of elasticity differences allows the system to 


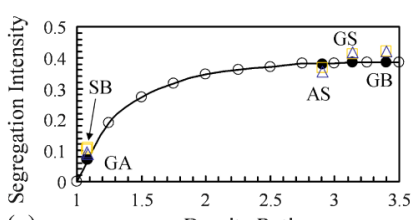

(a)

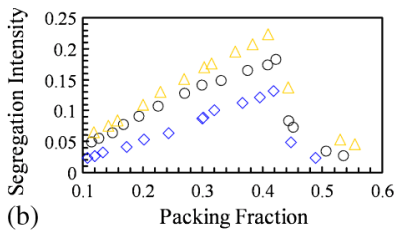

(b)
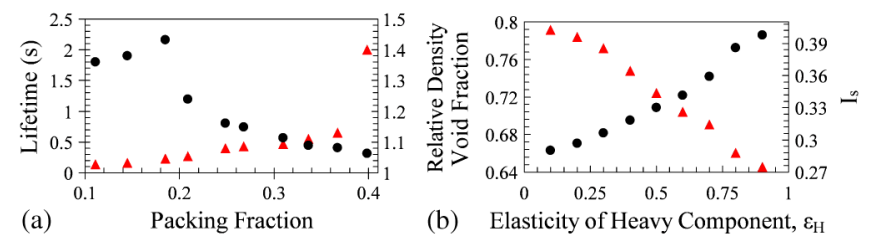

(b) Elasticity of Heavy Component, $\varepsilon_{\mathrm{H}}$
FIG. 2 (color online). (a) $I_{s}$ as a function of $\rho_{H} / \rho_{L}$ for $H=10$. Definitions of the symbols used are given in the main text. (b) Simulational data showing the variation of $I_{s}$ with packing fraction $\eta$ for purely inelasticity-driven segregation. Here, $\rho_{\alpha}=$ $\rho_{\beta}=2500 \mathrm{~kg} \mathrm{~m}^{-3}$ and values of $\epsilon_{\alpha \alpha} / \epsilon_{\beta \beta}$ are 4 (triangles), 2 (circles), and 1.5 (diamonds). In each case, $\epsilon_{\alpha \alpha}=0.95$.

exceed the plateau value of $I_{s}$. This may be explained by a reduced void space [39] in the lower bed for the case of more dissipative heavy particles, which will be more prone to condensation [40]. An example of the reduction in void space for increasingly dissipative particles-and the corresponding increase in segregation-may be seen in Fig. 3(a). Figure 2(a) shows data for a bed of resting height $H=10$. The increased packing fraction $\eta$ impacts the system in several noteworthy ways. First, the influence of the differing elasticities, although still present, is significantly reduced. Second, for purely density-driven segregation, we find an increased maximal $I_{s}$. Finally, the time taken for the system to attain its final segregated state is also found to increase. Having established that simulations provide an accurate representation of the experimental system, we now proceed to provide a more detailed analysis of these effects arising from variations in $\eta$. Simulational and experimental results show that, for a fixed excitation, $\eta$ varies with $H$ following the approximate form $\eta=\eta_{\max }[1-\exp (-(k H / \epsilon))]$, where $\eta_{\max }$ is the packing fraction at rest and $k$ is a constant ( $k \approx \frac{1}{6}$ for the current setup), allowing a range of $\eta$ to be investigated. Figure 2(b) illustrates the effect of $\eta$ on purely inelasticity-driven segregation. Significant segregation is observed over a wide range of $\eta$, implying that $\epsilon$ plays an important role in segregative processes for both dilute and moderately dense systems. Simulations using a variety of elasticity ratios demonstrate a similar trend in all cases, only differing significantly in the magnitudes of the observed $I_{s}$ values. In all cases, $I_{s}$ initially increases with $\eta$, before reaching a maximum and decreasing sharply. This behavior can be understood in terms of the theory of Brito et al. [41] regarding inelasticity-induced segregation. It is hypothesized in [41] that inelasticity-induced segregation occurs due to the formation of "cold droplets," regions of high density and low temperature around inelastic particles. The presence of such droplets is confirmed in our simulations. The excess mass surrounding a particle is defined as $\delta m=\int_{d}^{\infty} d r 2 \pi r\left[\tilde{\rho}_{\beta}-\tilde{\rho}_{\alpha}\right]$, where $\tilde{\rho}_{X}$ is the density surrounding a particle of species $X$. The increased effective weight of an inelastic particle allows it to overcome buoyancy forces and "sink" downwards, creating a degree
FIG. 3 (color online). (a) Variation with $\epsilon_{H}$ of the average void fraction (black circles) for the lower half of a system with $H=$ 2.5 and $\rho_{H} / \rho_{L}=4$ alongside the corresponding variation in $I_{s}$ (red triangles). (b) Typical lifespan (red triangles) and relative density (black circles) of "cold droplets" at varying packing fractions for $\rho_{\alpha}=\rho_{\beta}=2500$ and $\epsilon_{\alpha}=3 \epsilon_{\beta}=0.9$.

of segregation. However, as can be seen in Fig. 3(b), as $\eta \rightarrow \eta_{\max }, \delta m \rightarrow 0$, explaining the decrease in $I_{s}$ at large $\eta$. This also explains the absence of significant inelasticity effects on segregation observed in previous studies concerning high-density systems [42]. Conversely, as $\eta \rightarrow 0$, the droplets' typical lifespan tends to 0 as energy-injecting interactions with the system's base become increasingly prevalent $[43,44]$ and more free space becomes available for individual beads to separate from the clusters [45]. The combination of these factors gives an increased rate of droplet dispersal [45-48], hence the observed decrease in $I_{s}$.

In Fig. 4(a) we compare, for a range of packing fractions, the maximal degree of segregation achievable due solely to density differences (i.e., $\epsilon_{\alpha \alpha} / \epsilon_{\beta \beta}=1$ ) with the average time required for the system to achieve this value of $I_{s}$. Data
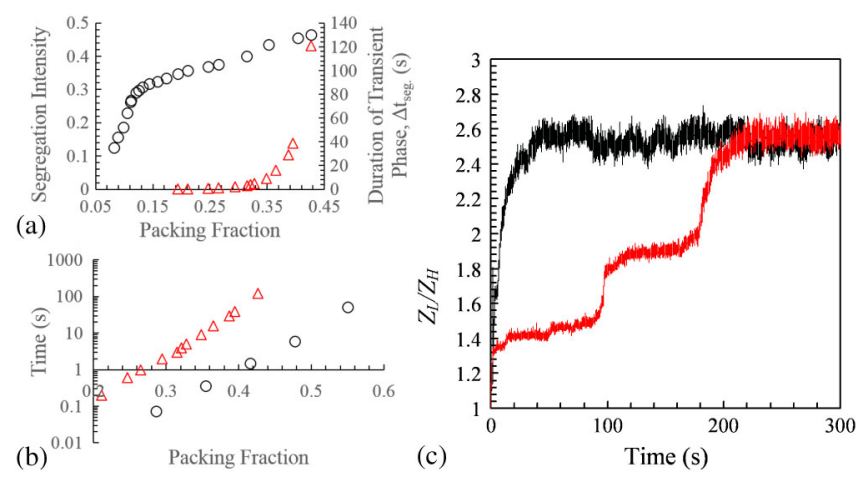

FIG. 4 (color online). (a) Variation with $\eta$ of the asymptotic upper limit of $I_{s}$ (circles) alongside the time taken for the system to reach this limit (triangles). Data correspond to simulations of purely density-driven segregation $\left(\epsilon_{\alpha \alpha} / \epsilon_{\beta \beta}=1\right)$. (b) Time taken for a simulated system to achieve steady-state segregation (triangles) and experimental single-particle rise time (circles) as a function of system density. (c) Time-evolving ratio of the centers of mass of light $\left(Z_{L}\right)$ and heavy $\left(Z_{H}\right)$ particles. $Z_{L} / Z_{S}$ gives an indication of the degree to which a system is segregated. The red line corresponds to a system with resting bed height $H=24.4$ driven with constant acceleration $\Gamma=17$. The black line represents a similar system whose driving varies as described in the main text. 
shown correspond to the case $\rho_{H} / \rho_{L}=5$, adequately high to ensure that the plateau value of $I_{S}$ is attained for all systems. As $\eta$ increases, segregation becomes more complete, but the rate at which this segregation occurs decreases considerably. The general increase in maximal $I_{s}$ with system density can be simply explained by the corresponding decrease in void space and increased dominance of buoyancy forces over diffusive processes within the system [49]. It is interesting to note that the sudden downturn in the trend at low densities occurs at the point where the system's mean free path is approximately equal to the particle diameter. Figure 4(b) shows the segregation rate data replotted on a logarithmic scale. In order to verify these results, additional experiments were conducted to measure the rise time [50], $\Delta t_{\text {rise }}$, of a single glass "intruder" in a bed of $N=500$ steel spheres. $\Delta t_{\text {rise }}$ is defined as the time taken for the intruder to travel from the base of the system to its free surface. The values shown correspond to the average over a number of such transits and are normalized by the dimensionless dynamic bed height $\tilde{H} / d$. Although $\Delta t_{\text {seg }}$ and $\Delta t_{\text {rise }}$ are not directly equivalent, the similarity of their general trends supports the validity of the simulational model. For relatively dilute systems, the final segregated state is achieved extremely rapidly, with $\Delta t_{\text {seg }} \lesssim 1 \mathrm{~s}$ [51]. As $\eta$ increases, however, $\Delta t_{\text {seg }}$ increases exponentially. Specifically, an increase of the form $C_{1} \exp \left(C_{2} \eta\right)$ is observed, with $C_{2} \approx 28$ for the case of the simulated $\Delta t_{\text {seg }}$ and $C_{2} \approx 24$ for the experimental $\Delta t_{\text {rise. The fact }}$ that, as $\eta$ increases, the maximal $I_{s}$ increases yet the rate of segregation decreases raises an interesting question: could a fully segregated system be produced in a less timeconsuming and more energy efficient manner by varying driving of the system? To test this hypothesis, simulations were conducted using an initial high- $A$ driving to rapidly produce partial segregation, before reducing $A$, allowing the system to relax into a denser state in which more complete segregation is achievable. Figure 4(c) shows examples of the time evolution of the relative positions of the vertical centers of mass of heavy and light species for the case of $\rho_{H} / \rho_{L}=5$ and $\epsilon_{\alpha \alpha} / \epsilon_{\beta \beta}=1$. The plots correspond to identical systems, differing only in their initial driving: the red line shows the case of a system driven uniformly at $f=70 \mathrm{~Hz}$ and $A_{1}=0.862 \mathrm{~mm}$, the black line a system driven at the same frequency, but with an initial amplitude $A_{2}=2 A_{1}$ which, after a period of $2 \mathrm{sec}$, is reduced to $A_{1}$, where it is held for the remainder of the run. This latter method of driving allows the system to reach maximal segregation considerably more rapidly than the former. The initial strong driving produces a relatively highly fluidized system allowing the rapid accession of a partially segregated state. Analysis of mean squared displacement also implies that the initially increased $A$ results in a relatively disordered, loose packing even after being reduced to $A_{1}$, allowing greater mobility [52] and hence a continued increase in segregation rate. This hypothesis may also explain why the steplike rise in $Z_{L} / Z_{H}$, for the single$A$ system is not observed in the varied- $A$ system; for the latter system, where a degree of fluidization is maintained, segregation is buoyancy driven, giving a relatively rapid, smooth increase in $I_{s}$. For the former system, a lack of fluidization means the condition for buoyancy is not fulfilled [53], segregation instead occurring through particle rearrangement, resulting in the sudden, discrete increases in $Z_{L} / Z_{H}$ observed. Although this steplike behavior is not particularly surprising [54,55], the strongly hysteretic nature of the system, and the resulting ability to exploit this hysteresis in order to produce markedly different segregative behaviors in identical systems, is striking. This observation is potentially of significance to industryfor industrial processes which require segregation, the use of varied driving may result in considerable savings in both time and energy. Although the evolution of the fixed- $A$ system is nondeterministic, repeated simulations show $\Delta t_{\text {seg }}$ to be consistently greater for this system than for the varied- $A$ system. On average, the time required to achieve maximal segregation is reduced by $\approx 80 \%$, while the necessary energy input to give the required $I_{s}$ is reduced by approximately a factor of 4 . Through further alterations to the manner of driving, it should be possible to produce even greater reductions in time and energy expenditure, making this a subject worthy of further research.

Conclusions.-The influence of system density on segregation due to differences in material density and inelasticity has been studied, producing the following key results: first, it is demonstrated that the effects of particle inelasticity persist to a significant degree over a wide range of system densities, becoming negligible only as the system approaches a nonfluidized state. This observation illustrates the importance of considering these effects both in industry and future research. Second, the maximal level of densitydriven segregation achievable is found to be limited by a system's void fraction. It is also demonstrated that the introduction of inelasticity effects can allow this upper limit to be exceeded. Third, it is found that increasing packing fraction leads to an exponential increase in the time required for a system to reach its maximally segregated state. Finally, and perhaps most strikingly, it is demonstrated that through adjustment driving strength, it is possible to significantly decrease the time and energy required to separate a binary system into its individual constituents. Such a finding clearly has potential industrial applications.

The authors would like to thank Professor Stefan Luding for his highly useful comments and discussion, as well as for access to the computer facilities on which the simulations in this Letter were performed. We gratefully acknowledge the financial support of the Hawkesworth Scholarship, kindly provided by the late Dr. Michael Hawkesworth. 
*windowsyule@gmail.com

[1] H. M. Jaeger, S. R. Nagel, and R. P. Behringer, Phys. Today 49, No. 4, 32, (1996).

[2] K. Ahmad and I. J. Smalley, Powder Technol. 8, 69 (1973).

[3] S. C. Yang, Powder Technol. 164, 65 (2006).

[4] A. Rosato, K. J. Strandburg, F. Prinz, and R. H. Swendsen, Phys. Rev. Lett. 58, 1038 (1987).

[5] C. Li, Z. Zhou, R. Zou, D. Pinson, and A. Yu, AIP Conf. Proc. 1542, 767 (2013).

[6] D. Serero, I. Goldhirsch, S. H. Noskowicz, and M.-L.Tan, J. Fluid Mech. 554, 237 (2006).

[7] R. Brito and R. Soto, Eur. Phys. J. Spec. Top. 179, 207 (2009).

[8] F. J. Muzzio, T. Shinbrot, and B. J. Glasser, Powder Technol. 124, 1 (2002).

[9] A. R. Thornton, D. Krijgsman, A. te Voortwis, V. Ogarko, S. Luding, R. Fransen, S. Gonzalez, O. Bokhove, O. Imole, and T. Weinhart, in DEM 6: Proceedings of the 6th International Conference on Discrete Element Methods and Related Techniques, 2013 (Colorado School of Mines, Colorado, 2013), p. 393.

[10] A. R. Thornton, T. Weinhart, S. Luding, and O. Bokhove, Int. J. Mod. Phys. C 23, 1240014 (2012).

[11] A. R. Thornton, T. Weinhart, V. Ogarko, and S. Luding, Comput. Methods Mater. Sci. 13, 197 (2013).

[12] MercuryDPM.org.

[13] N. Burtally, P. J. King, and M. R. Swift, Science 295, 1877 (2002).

[14] M. E. Möbius, X. Cheng, G. S. Karczmar, S. R. Nagel, and H. M. Jaeger, Phys. Rev. Lett. 93, 198001 (2004).

[15] X. Yan, Q. Shi, M. Hou, K. Lu, and C. K. Chan, Phys. Rev. Lett. 91, 014302 (2003).

[16] The strong agreement between experimental results and simulations, in which air effects were deliberately omitted, also implies an absence of air effects in the system.

[17] C. Zeilstra, M. A. van der Hoef, and J. A. M. Kuipers, Phys. Rev. E 77, 031309 (2008).

[18] P. Eshuis, K. van der Weele, D. van der Meer, and D. Lohse, Phys. Rev. Lett. 95, 258001 (2005).

[19] J.-C. Géminard and C. Laroche, Phys. Rev. E 68, 031305 (2003).

[20] S. Luding, H. J. Herrmann, and A. Blumen, Phys. Rev. E 50, 3100 (1994).

[21] J. S. van Zon, J. Kreft, D. I. Goldman, D. Miracle, J. B. Swift, and H. L. Swinney, Phys. Rev. E 70, 040301(R) (2004).

[22] D. I. Goldman, M. D. Shattuck, S. J. Moon, J. B. Swift, and H. L. Swinney, Phys. Rev. Lett. 90, 104302 (2003).

[23] J. B. Knight, Phys. Rev. E 55, 6016 (1997).

[24] D. J. Parker, R. N. Forster, P. Fowles, and P. S. Takhar, Nucl. Instrum. Methods Phys. Res., Sect. A 477, 540 (2002).

[25] R. D. Wildman, J. M. Huntley, J.-P. Hansen, D. J. Parker, and D. A. Allen, Phys. Rev. E 62, 3826 (2000).

[26] R. D. Wildman and D. J. Parker, Phys. Rev. Lett. 88, 064301 (2002).

[27] R. D. Wildman and J. M. Huntley, Phys. Fluids 15, 3090 (2003).

[28] K. Feitosa and N. Menon, Phys. Rev. Lett. 88, 198301 (2002).

[29] This determination of $\epsilon_{\alpha \beta}$ arises naturally from the springdashpot model of particle restitution [30-32]. Despite the simplicity of this assumption, excellent agreement with experimental data is found across all data sets.

[30] P. A. Cundall and O. D. L. Strack, Geotechnique 29, 47 (1979).

[31] S. Luding, Granular Matter 10, 235 (2008).

[32] T. Weinhart, A. R. Thornton, S. Luding, and O. Bokhove, Granular Matter 14, 531 (2012).

[33] The value of $\epsilon_{w}$ used in the simulations corresponds to the experimentally measured mean energy loss for collisions between a glass particle and the system sidewall, averaged over a large number of such collisions. The specific value of $\epsilon_{w}$ was found not to significantly affect the system behavior so long as it remained sufficiently high to suppress convection [34].

[34] C. R. K. Windows-Yule, N. Rivas, and D. J. Parker, Phys. Rev. Lett. 111, 038001 (2013).

[35] See Supplemental Material at http://link.aps.org/ supplemental/10.1103/PhysRevLett.112.098001 for full details of the MERCURYDPM code.

[36] D. V. Khakhar, J. J. McCarthy, T. Shinbrot, and J. M. Ottino, Phys. Fluids 9, 31 (1997).

[37] G. V. Middleton and M. Hampton, Subaqueous Sediment Transport and Deposition by Sediment Gravity Flows, edited by D. J. Stanley and D. J. P. Swift (Wiley, New York, 1976), p. 197.

[38] S. B. Savage and C. K. K. Lun, J. Fluid Mech. 189, 311 (1988).

[39] J. S. Olafsen and J. S. Urbach, Phys. Rev. Lett. 81, 4369 (1998).

[40] D. C. Hong, P. V. Quinn, and S. Luding, Phys. Rev. Lett. 86, 3423 (2001).

[41] R. Brito, H. Enriquez, S. Godoy, and R. Soto, Phys. Rev. E 77, 061301, (2008).

[42] Q. Shi, G. Sun, M. Hou, and K. Lu, Phys. Rev. E 75, 061302 (2007).

[43] J. S. van Zon and F. C. MacKintosh, Phys. Rev. Lett. 93, 038001 (2004).

[44] J.S. van Zon and F. C. MacKintosh, Phys. Rev. E 72, 051301 (2005).

[45] K. L. Gavrilov, Phys. Rev. E 58, 2107 (1998).

[46] A. Kudrolli and J. Henry, Phys. Rev. E 62, R1489 (2000).

[47] A. Kudrolli, M. Wolpert, and J. P. Gollub, Phys. Rev. Lett. 78, 1383 (1997).

[48] B. J. McCoy and G. Madras, Phys. Rev. E 70, 051311 (2004).

[49] D. V. Khakhar, J. J. McCarthy, and J. M. Ottino, Phys. Fluids 9, 3600 (1997).

[50] L. Vanel, A. D. Rosato, and R. N. Dave, Phys. Rev. Lett. 78, 1255 (1997).

[51] Although the PEPT technique is unsuitable for measuring the temporal evolution of $I_{s}$, the surprising rapidity of this segregation has been confirmed through visual observations of the experimental system.

[52] A. D. Rosato and D. Yacoub, Powder Technol. 109, 255 (2000).

[53] D. A. Huerta and J. C. Ruiz-Suarez, Phys. Rev. Lett. 92, 114301 (2004).

[54] S. Dippel and S. Luding, J. Phys. I (France) 5, 1527 (1995).

[55] J. Duran, J. Rajchenbach, and E. Clément, Phys. Rev. Lett. 70, 2431 (1993). 